\title{
Nanofabrication Technologies for All
}

\author{
Hiroyuki Akinaga $^{* \dagger}$ \\ National Institute of Advanced Industrial Science and Technology, \\ Central 5, 1-1-1 Higashi, Tsukuba, Ibaraki 305-8565, Japan
}

(Received July 14, 2019; accepted July 18, 2019)

Keywords: nanofabrication, nanotechnology, SDGs Goal 9, on-demand manufacturing, open user foundry

Advanced nanofabrication technologies have fostered a manufacturing revolution in various industrial fields. Technological capabilities previously were owned only by researchers and large companies, but have become easily accessible, thanks to IoT-related technologies. Such easy accessibility is imperative to achieve the targets of Sustainable Development Goals (SDGs) Goal 9. A brief historical review and difficult challenges with their prospects are presented in order to promote the further advancement of nanofabrication not only for SDGs Goal 9 but also for other SDGs Goals.

\section{Role of Nanofabrication}

Manufacturing is certainly a principal driver of sustainable and economic development of our society, as claimed in Sustainable Development Goals (SDGs) Goal 9. ${ }^{(1,2)}$ The progressive development of fabrication technologies has brought about a manufacturing revolution in various industrial fields. More specifically, elemental technologies of thin film deposition, lithography, etching, cleaning, and database-driven design are being developed for the nanoscale. Nanotechnology in fabrication processes is a fundamental and indispensable tool to spur the development of emerging materials and devices, such as transistors, memories, sensors, and microactuators.

\section{Brief Historical Review of Nanofabrication}

Figure 1 shows a brief history and the prospect of nanoscale fabrication technologies. In 2000, President William J. Clinton (USA) advocated the National Nanotechnology Initiative, and stated, "Some of our research goals may take 20 or more years to achieve, but that is precisely why there is an important role for the federal government." The initiative supports long-term nanoscale research and development leading to potential breakthroughs in areas such as manufacturing, nanoelectronics, environment and energy, biotechnology, agriculture, and information technology. ${ }^{(3,4)}$

\footnotetext{
*Corresponding author: e-mail: akinaga.hiro@aist.go.jp
}

†ORCID: http://orcid.org/0000-0002-5521-3148

https://doi.org/10.18494/SAM.2019.2521 


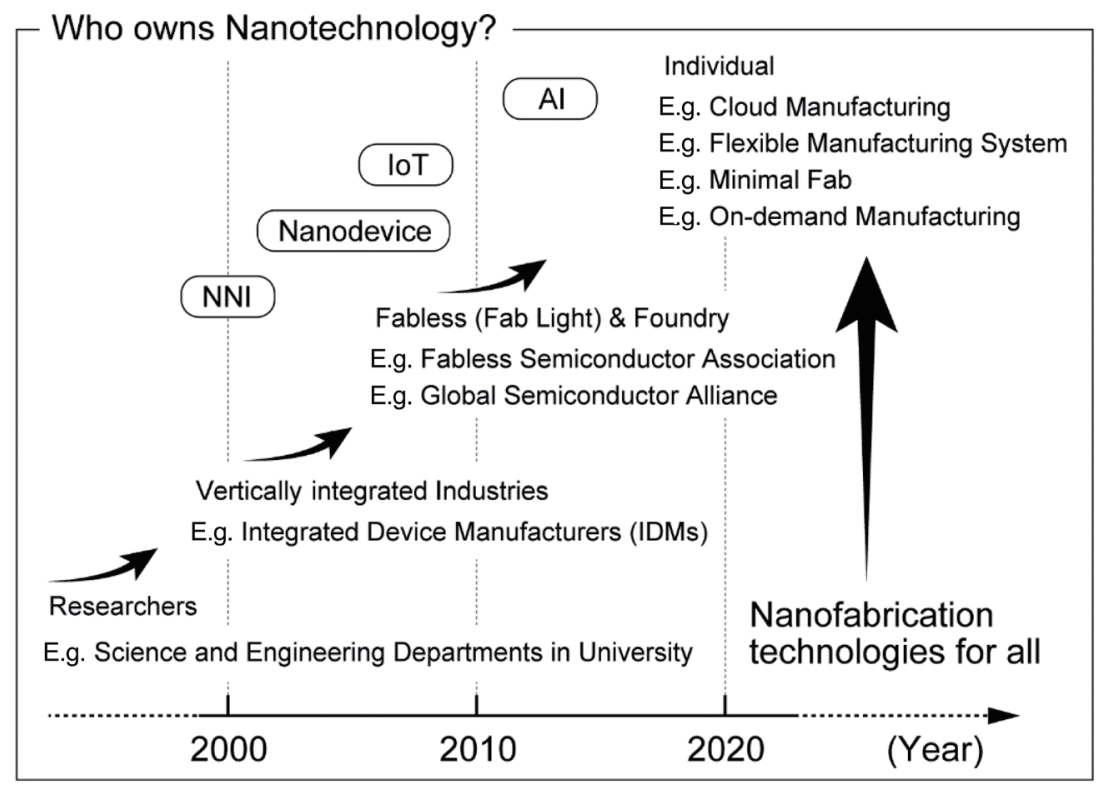

Fig. 1. Who owns nanotechnology? Cloud manufacturing will spread widely by making access to nanotechnologies easy.

According to the International Technology Roadmap for Semiconductors (ITRS) 2005 Edition, ${ }^{(5)}$ the actual and annual worldwide wafer production technology capacity distributions have moved into the sub-100 nm era. Note that "nanoscale" is defined as the size range from approximately 1 to $100 \mathrm{~nm}$. In this range, one can realize nano-enabled devices in which the material elements or assembly of such elements exhibit performance or function possible only with nanotechnology. ${ }^{(6)}$ The sub-100 nm space is, so to speak, the "nanoworld" where typical nano-originated physical properties, such as quantum phenomena, meet practicality. Actually, International Organization for Standardization (ISO) established Technical Committee (TC) 229 (Nanotechnologies) in 2005, and International Electrotechnical Comission (IEC) launched TC 113 (Nanotechnology for electrotechnical products and systems) in 2006. These facts are evidence that true nanodevices appeared in our market and society in around 2005. Our society was changed with the advent of the nanoworld. For example, the Internet of Things (IoT) was born sometime between 2008 and 2009. The number of connected devices per human exceeded 1. ${ }^{(7)}$ International Roadmap for Devices and Systems (IRDS) released the figure of novel computing paradigms showing that such paradigms have been rebooted progressively to accelerate novel applications, such as artificial intelligence (AI) and big data. ${ }^{(8)}$

\section{Prospects and Difficult Challenges}

The development of IoT will be returning nanofabrication technologies to our hands. For example, service-oriented technologies enable customers to utilize a new manufacturing paradigm, cloud manufacturing. ${ }^{(9,10)}$ The pay-by-use and pay-as-you-go scenarios will revolutionize user manufacturing. ${ }^{(11)}$ Even without a cleanroom, one can develop semiconductor 


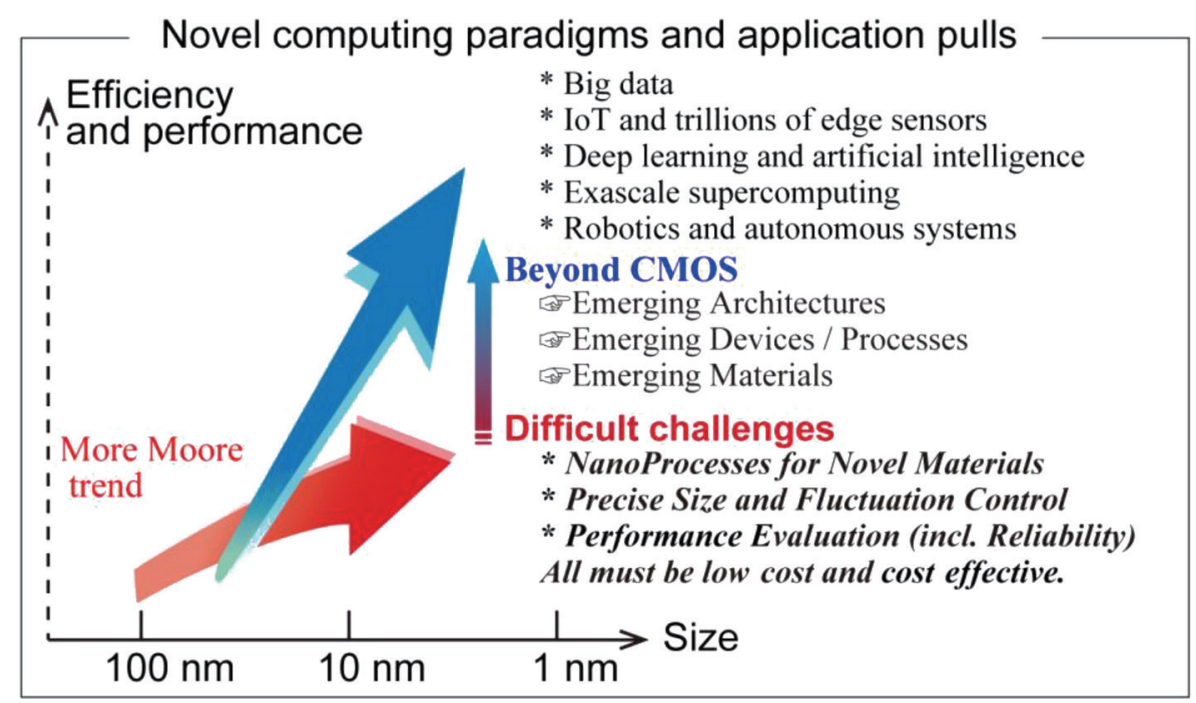

Fig. 2. (Color online) Novel computing paradigms and application pulls. The difficult challenges are shown. Nanofabrication technologies must be continuously developed for the promotion of these pulls.

devices in the Minimal Fab System. ${ }^{(12)}$ The on-demand manufacturability will expand the scope of the beneficiaries, followed by a maker movement. ${ }^{(13)}$ The near-future prospect of nanofabrication technologies is summarized in Fig. 1. Here, open user foundries are the most practical platform for us to get a chance to use advanced nanofabrication facilities. ${ }^{(14)}$ The facilities with well-organized know-how will be strong tools for realizing our own idea without the high-cost investment of cutting-edge equipment. From the technological viewpoint, the difficult challenges will be low-cost and high-precision nanoprocessing technologies for various emerging materials. Process evaluation, such as morphological observation and measurement, and performance evaluation, such as reliability and durability tests, should be continuously developed. Operando measurements and analyses will also play crucial roles in flexible manufacturing for practical applications. These challenges are shown in Fig. 2. Making international access to the above-mentioned user foundry easy via IoT networks will certainly contribute to attaining SDGs Goal 9. I sincerely hope that this special issue focusing on Advanced Nanofabrication Processes for Emerging Devices and Materials will nurture the open user foundry as an active pathway for all users to promote the advancement of nanofabrication not only for SDGs Goal 9 but also for other SDGs Goals.

\section{References}

1 Transforming our world: the 2030 agenda for sustainable development (Internet). New York: United Nations. (accessed 14 July 2019). https://sustainabledevelopment.un.org/post2015/transformingourworld/

2 Sustainable development goal 9; Build resilient infrastructure, promote inclusive and sustainable industrialization and foster innovation (Internet). New York: United Nations. (accessed 14 July 2019). https:// sustainabledevelopment.un.org/sdg9/

3 National Nanotechnology Initiative: The Initiative and its Implementation Plan, National Science and Technology Council, Committee on Technology, Subcommittee on Nanoscale Science, Engineering and Technology (July 2000). 
4 Exploring the Wonders of the Nano-World: A Conversation with Dan Herr (accessed 14 July 2019). https://stories-from-the-nni.simplecast.com/episodes/dan-herr/ https://youtu.be/qT3k51Hae2M

5 Technology Cycle Timing Compared to Actual Wafer Production Technology Capacity Distribution, Fig. 4 in International Technology Roadmap for Semiconductors (ITRS) 2005 Edition: Executive Summary. (accessed 14 July 2019). http://www.itrs2.net/

6 These definitions were issued by the International Organization for Standardization (ISO) and International Electrotechnical Commission (IEC). Both ISO and IEC organized technical committees (TCs) in the field of nanotechnology, that is, TC229 (Nanotechnologies) and TC113 (Nanotechnology for electrotechnical products and systems), respectively.

ISO TC 229 (accessed 14 July 2019). https://www.iso.org/committee/381983.html

IEC TC 113 (accessed 14 July 2019). https://www.iec.ch/dyn/www/f?p=103:7:1356605119205::::FSP_ORG_ ID:1315

7 D. Evans: "The Internet of Things: How the Next Evolution of the Internet Is Changing Everything" (accessed 14 July 2019). https://www.cisco.com/c/dam/en_us/about/ac79/docs/innov/IoT_IBSG_0411FINAL.pdf

8 The figure is reproduced with modification of Fig. BC1.1, "Relationship of More Moore, Beyond CMOS, and Novel Computing Paradigms and Applications" in Beyond CMOS chapter, International Roadmap for Devices and Systems (IRDS), 2018 Update. (accessed 14 July 2019). https://irds.ieee.org/

9 F. Tao, Y. Cheng, L. D. Xu, L. Zhang, and B. H. Li: IEEE Trans. Ind. Inf. 10 (2014) 1435. https://doi. org/10.1109/TII.2014.2306383

10 L. Zhang, Y. Luo, F. Tao, B. H. Li, L. Ren, X. Zhang, H. Guo, Y. Cheng, A. Hu, and Y. Liu: Enterp. Inf. Syst. 8 (2011) 167. https://doi.org/10.1080/17517575.2012.683812.

11 X. Xu: Rob. Comput.-Integr. Manuf. 28 (2012) 75. https://doi.org/10.1016/j.rcim.2011.07.002

12 S. Khumpuang and S. Hara: Int. Conf. IC Design and Technology (ICICDT), Ho Chi Minh City, Vietnam (2016). https://doi.org/10.1109/ICICDT.2016.7542054

13 D. Dougherty: "We are makers” (accessed 14 July 2019). https://www.ted.com/talks/dale_dougherty_we_are_ makers

14 H. Akinaga: Synthesiology 7 (2014) 1.

\section{About the Author}

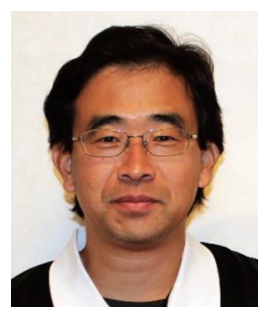

Hiroyuki Akinaga received his B.E., M.E., and Ph.D. degrees from the University of Tsukuba, Ibaraki, Japan, in 1987, 1989, and 1992, respectively. $\mathrm{He}$ is currently a senior research manager at the Nanoelectronics Research Institute, National Institute of Advanced Industrial Science and Technology, and the deputy research supervisor of the project of scientific innovation for energy harvesting technology, Japan Science and Technology Agency. His current interests include nanoelectronics and open innovation platforms. 